\title{
Broadband single cell impedance spectroscopy using maximum length sequences: theoretical analysis and practical considerations
}

\author{
Tao Sun, Shady Gawad, Catia Bernabini, Nicolas G Green \\ and Hywel Morgan \\ Nanoscale Systems Integration Group, School of Electronics and Computer Science, \\ University of Southampton, Southampton SO17 1BJ, UK \\ E-mail: ts04r@ecs.soton.ac.uk and hm@ecs.soton.ac.uk
}

Received 1 March 2007, in final form 6 June 2007

Published 20 July 2007

Online at stacks.iop.org/MST/18/2859

\begin{abstract}
Measurements of the dielectric (or impedance) properties of cells can be used as a general characterization and diagnostic tool. In this paper, we describe a novel impedance spectroscopy technique for the analysis of single biological cells in suspension. The technique uses maximum length sequences (MLS) for periodic excitation signal in a microfluidic impedance cytometer. The method allows multi-frequency single cell impedance measurements to be made in a short time period (ms). Spectral information is obtained in the frequency domain by applying a fast M-sequence transform (FMT) and fast Fourier transform (FFT) to the time domain response. Theoretically, the impedance is determined from the transfer function of the system when the MLS is a current excitation. The order of the MLS and sampling rate of A/D conversion are two factors that determine the bandwidth and spectral accuracy of the technique. Experimentally, the applicability of the technique is demonstrated by characterizing the impedance spectrum of red blood cells (RBCs) in a microfluidic cytometer. The impedance is measured within $1 \mathrm{~ms}$ at 512 discrete frequencies, evenly distributed in the range from $976.56 \mathrm{~Hz}$ to $500 \mathrm{kHz}$. The measured spectrum shows good agreement with simulations.
\end{abstract}

Keywords: impedance spectroscopy, single cell analysis, maximum length sequences, microfluidic cytometer

(Some figures in this article are in colour only in the electronic version)

\section{Introduction}

Dielectric spectroscopy has been used to characterize heterogeneous biological systems since Höber's earliest electrical conductivity measurements on erythrocytes in 1910s [1-3]. During the last century dielectric spectroscopy became an important tool in the study of the passive properties of biological cells and tissues, and was widely used by scientists such as Fricke [4-6] and Curtis [7], Cole [8-10] and Cole
[11, 12], Schwan [13, 14] and Foster [15], Pethig [16], Zimmermann [17], Hanai [18] and Asami [19]. Generally, the dielectric analysis of single cells has been performed using ac electrokinetic methods such as dielectrophoresis (DEP) [20-22], travelling wave dielectrophoresis (twDEP) $[23,24]$ and electrorotation (ROT) [25, 26]. Analysis of cell suspensions is performed using electrical impedance spectroscopic (EIS) methods [27-30], including time domain dielectric spectroscopy (TDDS) [31]. 


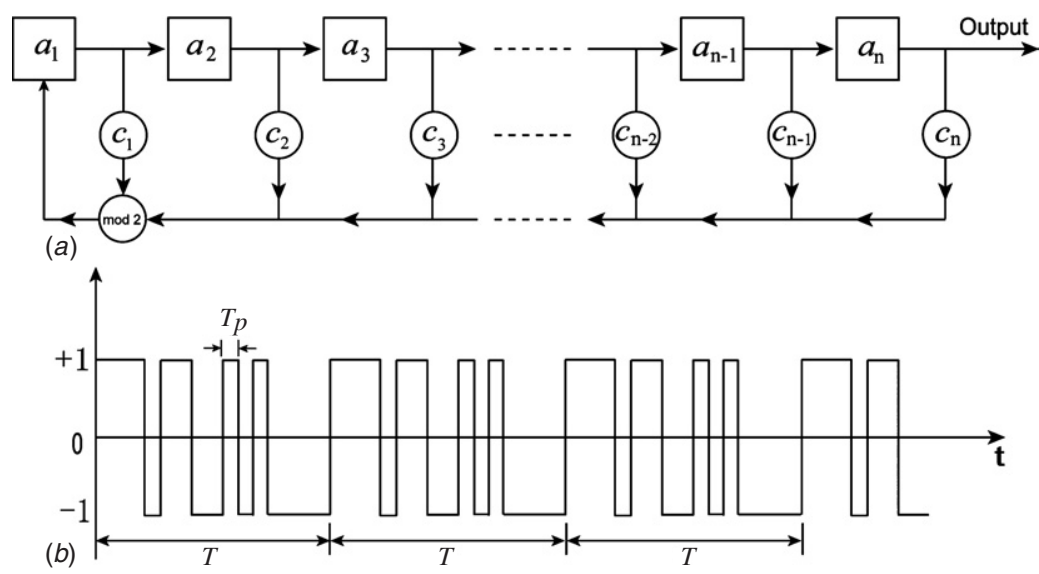

Figure 1. (a) Structure of the digital shift registers, in series with feedback coefficients, used to recurrently generate the maximum length sequences (MLS). (b) A typical analogue bipolar MLS in the time domain.

In recent years, microfluidic cytometers [32-36] have been developed that use impedance spectroscopy as a fast and label-free technique to characterize the impedance properties of single particles. Thin planar microelectrodes are fabricated inside a microchannel, along which the cells flow. The electrodes are energized by an ac voltage at one or more discrete frequencies and the impedance of the cell is determined from the current response of the system. In order to maximize the sensitivity, a differential impedance measurement is implemented, with the current amplified by a trans-impedance amplifier. Lock-in amplifiers are used to demodulate the signals at each discrete frequency. Details of the system have been published previously [32, 34-36]. However, a major drawback of the discrete frequency ac impedance measurement system is that the number of measurement frequencies is limited because the measured signal at every discrete frequency requires to be demodulated by one corresponding lock-in amplifier.

To overcome this problem, we propose a new technique which uses maximum length sequence (MLS) analysis to characterize the transfer function of the system and thereby extract the impedance or the dielectric properties of single cells over a wide frequency band in a short time window. MLS is a pseudo random binary sequence (PRBS) and has a white-noise-like power spectrum with the energy uniformly spread over a wide frequency range, enabling high speed multi-frequency measurements. It has been widely used in the field of audio engineering and architectural acoustics. Davies [37] in 1966 introduced MLS as a method that could measure the impulse response of a linear time invariant (LTI) system, and since then a number of papers have described the computational algorithms [38-44] and practical applications [45-48]. The use of MLS in research fields other than acoustics has been gradually recognized only in recent years [49-54]. However, there is little activity in its use for impedance spectroscopy other than the early paper of Schneider [50] who first proposed that the MLS could be used to characterize the bio-impedance of long bone fractures, but detailed theoretical analysis or experimental data was not presented. In this paper, we review the basic principles of the MLS measurement technique and the instrumentation of the measurement system. Based on previous work [55], we develop a theoretical model for the impedance spectrum of a single-shelled dielectric object (a cell) in suspension, which is obtained by using MLS technique and the analysis is performed both in the time and frequency domains. Finally, we experimentally prove that the MLS technique can be used to characterize the impedance spectrum of red blood cells (RBCs) using a microfabricated impedance cytometer.

\section{Principle of MLS}

As a PRBS, the MLS signal is generated by an $n$-stage digital shift register with feedback coefficient control, conforming to the linear recurrence:

$$
a_{n}=\left(\sum_{i=1}^{n} c_{i} a_{i}\right) \bmod (2)
$$

As shown in figure 1(a), each newly generated element comes from register $a_{n}$, which is dependent on the states of all the registers and the feedback coefficients. At each time unit, the elements in each register are shifted one to the right. The sum is calculated with modulus 2 or exclusive-OR operation. The feedback coefficients $c_{i}$ are described by a primitive polynomial:

$$
f(x)=1+\sum_{i=1}^{n} c_{i} x^{i}
$$

With a given initial state of the shift registers (except all zeros), equations (1) and (2) allow a specific MLS to be generated. In the practical measurements, the 1 and 0 states are often mapped onto -1 and +1 levels, respectively, producing a bipolar sequence symmetrical about zero. A typical bipolar MLS is shown in figure $1(b)$. The duration of every element in one MLS is represented by $T_{p}$ and the whole period of one sequence is given by $T=L \times T_{p}$. Here, $L$ is the length of one MLS given by $L=2^{n}-1$, with $n$ denoting the order of the sequence and also the number of digital shift registers.

The MLS technique is based on calculating the crosscorrelation function between the input MLS signal and the output response of a system, giving the periodic impulse response (PIR) of the system [42]. The normalized impulse response of the system is given by [43]

$$
\mathbf{h}=\frac{1}{L+1} \mathbf{M Y}
$$


where vectors $\mathbf{h}$ and $\mathbf{Y}$ are the vectors of $L$ elements corresponding to the impulse response and the output response of the system, respectively. $\mathbf{M}$ is the right-shifted MLS matrix of dimension $L \times L$, containing the circular delayed versions of the sequence, which can be factorized by

$$
\mathbf{M}=\mathbf{P}_{\mathrm{L}} \mathbf{H} \mathbf{P}_{\mathrm{S}}
$$

where $\mathbf{P}_{\mathrm{L}}$ and $\mathbf{P}_{\mathrm{S}}$ are the permutation matrices of dimensions of $L \times(L+1)$ and $(L+1) \times L$, respectively. The matrix $\mathbf{H}$ is the Sylvester-type Hadamard matrix of dimension of $(L+1) \times(L+1)$. Equation (4) provides an efficient and fast way to derive the cross-correlation function between the MLS excitation signal and the output response of the system. In the literature, this is called the fast M-sequence transform (FMT) [38]. It is based on the fast Hadamard transform (FHT), which is more efficient than the fast Fourier transform (FFT), consisting of only additions and subtractions. From signal processing theory [56], the impulse response of the system in the time domain is equivalent to the transfer function of the system in the frequency domain. Therefore, the Fourier transform of the impulse response (obtained using MLS) gives the transfer function of the system.

A schematic diagram of the MLS measurement system is illustrated in figure 2(a). The digital MLS signal is generated by custom-designed software, programmed in MATLAB ${ }^{\mathrm{TM}}$ (Mathworks, Inc., USA). After D/A conversion, the analogue bipolar MLS is applied to a linear time invariant (LTI) system. After low-pass filtering (LPF), the response of the system in the continuous time domain is sampled into digital form by an A/D converter. The clock rate of the MLS generator is the same as the A/D sampling rate. The sampled response is converted into the impulse response using the FMT. Finally, the FFT gives the transfer function of the system in the frequency domain.

\section{Theoretical analysis}

The theoretical analysis is used to derive the impedance spectrum of the system shown in figure $2(b)$, following excitation by an MLS signal. The equivalent electrical circuit of a single cell in suspension is also shown in figure $2(b)$ and illustrates the link between the impedance and the transfer function of the system. In this simple analysis, the effect of the electrical double layer is not included; for a full equivalent circuit model see [30]. The geometrical and dielectric parameters of the cell are shown in figure 2(c). Since MLS is a time-varying signal, the output response of the system under MLS excitation is a time-dependent signal. Therefore, the first step in the theoretical analysis is to determine the response of the system in the time domain. FMT and FFT are then used to obtain the spectrum in the frequency domain. The analysis is based on the following assumptions:

(a) In order to directly associate the impedance with the transfer function of the system, the excitation MLS signal is assumed to be a current, rather than voltage (figure 2(b)). Therefore, the transfer function is directly the impedance of the system given by

$$
H_{\text {sys }}(s)=\frac{V_{\text {sys }}(s)}{I_{\mathrm{MLS}}(s)}=\tilde{Z}_{\text {mix }}(s)
$$

where $H_{\text {sys }}(s)$ and $\tilde{Z}_{\text {mix }}(s)$ are the transfer function and the equivalent complex impedance of the model system, (a)

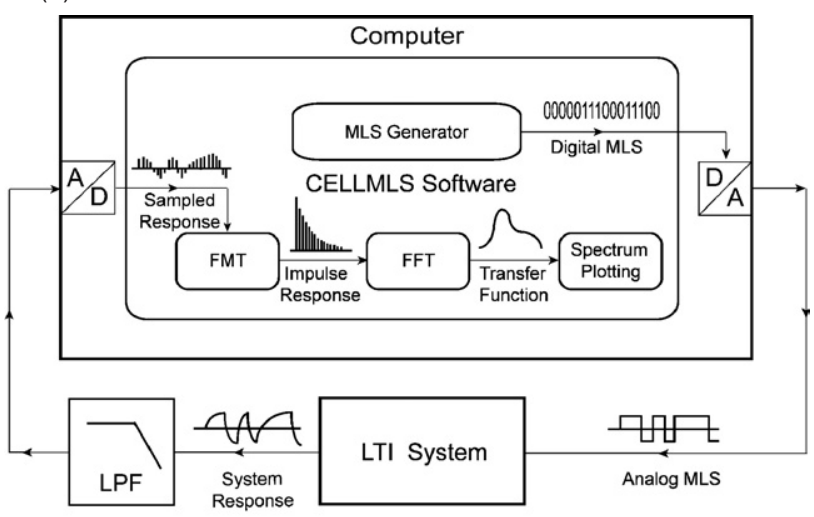

(b)

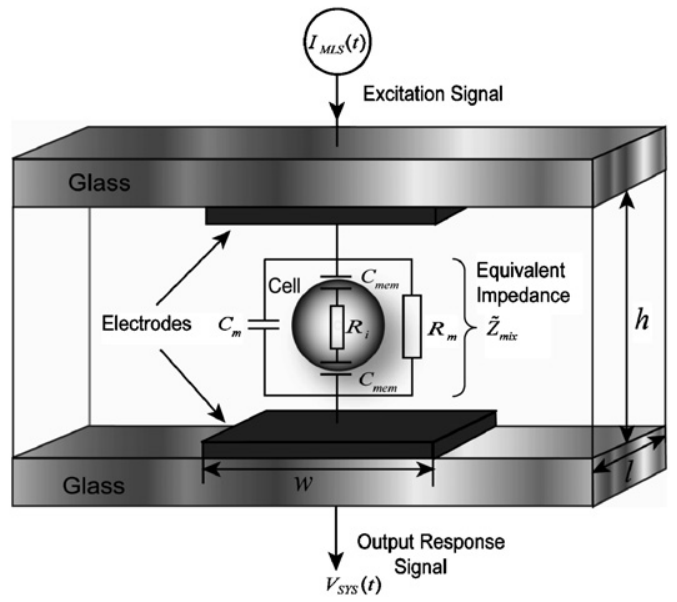

(c)

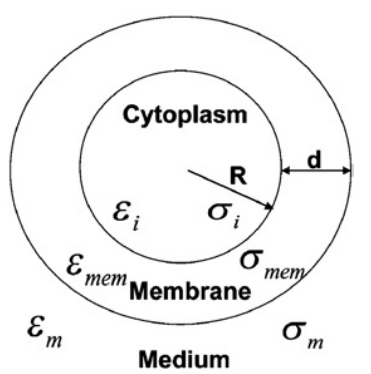

Figure 2. (a) Typical MLS measurement system set-up showing the MLS generation and processing software and the data flow path. (b) Diagram of the impedance cytometer with a single (static) cell in the centre, approximating a linear time invariant (LTI) system. The equivalent circuit model for a cell in suspension is also shown. $R_{m}$ and $C_{m}$ are the resistance and capacitance of the suspending medium, $C_{\text {mem }}$ is the capacitance of the cell membrane and $R_{i}$ is the resistance of the cytoplasm. The two electrodes have width $w$, length $l$ and the height of the channel is $h$. (c) The single-shelled cell model with the dielectric parameters of the different layers. $\varepsilon$ and $\sigma$ refer to the permittivity and conductivity, respectively.

respectively. $I_{\mathrm{MLS}}(s)$ is the MLS current excitation signal. $V_{\text {sys }}(s)$ is the voltage output response of the model system. All four terms are expressed in the frequency domain ( $s$-domain, $s=\mathrm{j} \omega$ and $\mathrm{j}^{2}=-1, \omega$ is the angular frequency).

(b) The MLS technique is generally used to characterize a linear time invariant (LTI) system. Therefore, we assume that the cell is stationary during the time of measurement. In a practical system cells flow across the measurement 
electrodes one by one. This assumption is only valid if the transit time of the cell across the electrodes is much shorter than the period of the MLS. For example, using a $1 \mathrm{MHz}$ sampling rate with a 10th-order MLS, the period of the MLS is approximately $1 \mathrm{~ms}$. For more than ten sequences to be applied to a cell, it must cross a $20 \mu \mathrm{m}$ wide electrode with a velocity of less than $2 \mu \mathrm{m} \mathrm{ms}^{-1}$ (or $2 \mathrm{~mm} \mathrm{~s}^{-1}$ ).

(c) The cell is located in the centre of the electric fields, midway between the two parallel facing electrodes as shown in figure $2(b)$. In this region, the electric field distribution is quasi-homogeneous [58], thus minimizing the influence of any fringing field on the impedance. Maxwell's mixture equation can be used to describe the dielectric behaviour of the particle in suspension.

\subsection{Time domain analysis}

For dilute suspensions (low volume fraction) of particles, the dielectric properties are well described by Maxwell's mixture equation. In a previous paper, we developed an analytical method to transform Maxwell's mixture equation from the frequency domain to the time domain using the Laplace transform [55]. Following this method, the expression for the complex conductivity $\tilde{\sigma}_{\text {mix }}(s)$, of a single-shelled spherical cell in suspension in the $s$-domain, is [55]

$$
\tilde{\sigma}_{\text {mix }}(s)=\frac{\lambda_{1} s^{3}+\lambda_{2} s^{2}+\lambda_{3} s+\lambda_{4}}{b_{1} s^{2}+b_{2} s+b_{3}} .
$$

The coefficients $\lambda_{i}$ and $b_{i}$ in equation (6) are given in the appendix.

The complex impedance of a cell in suspension, $\tilde{Z}_{\text {mix }}(s)$, is calculated from the complex conductivity of the system, provided the geometric cell constant $\kappa$ is known:

$$
\tilde{Z}_{\text {mix }}(s)=\frac{1}{\tilde{\sigma}_{\text {mix }}(s) l \kappa}
$$

where $l$ is the length of the electrode (see figure $2(b)$ ). For the micro-cytometer the calculation of the cell constant takes into account the fringing field $[30,32,58]$.

In the continuous time domain ( $t$-domain) the bipolar MLS current signal can be expressed using the superposition of unit step functions $\left(U_{0}(t)\right)$ :

$$
I_{\mathrm{MLS}}(t)=U_{0}(t)+2 \sum_{i=1}^{m}(-1)^{i} U_{0}\left(t-t_{i}\right) .
$$

Equation (8) is for the MLS signal with initial state +1 ; $t_{i}$ is when the polarity of the signal changes (from +1 to -1 and vice versa). The case for an MLS starting with -1 is similar.

Applying the Laplace transform to equation (8), the expression for the MLS in the frequency domain (s-domain) is

$$
I_{\mathrm{MLS}}(s)=\frac{1}{s}\left(1+2 \sum_{i=1}^{m}(-1)^{i} \mathrm{e}^{-s t_{i}}\right) .
$$

Combining equations (5), (7) and (9) gives the output response of the system in the $s$-domain:

$$
\begin{aligned}
V_{\mathrm{sys}}(s) & =I_{\mathrm{MLS}}(s) \tilde{Z}_{\mathrm{mix}}(s) \\
& =\frac{1}{s \tilde{\sigma}_{\text {mix }}(s) l_{\kappa}}\left(1+2 \sum_{i=1}^{m}(-1)^{i} \mathrm{e}^{-s t_{i}}\right) .
\end{aligned}
$$

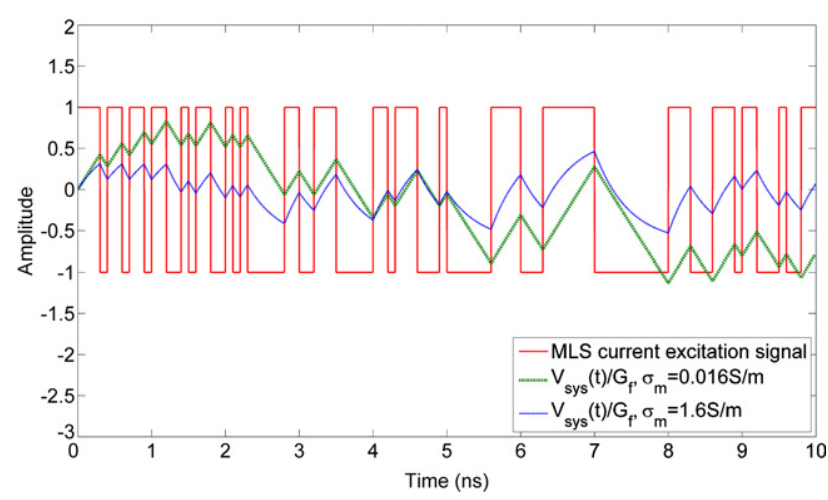

Figure 3. Time-dependent output responses of the system $V_{\text {sys }}(t)$ (divided by scale factor $\left.G_{f}\right)$ in low $\left(\sigma_{m}=0.016 \mathrm{~S} \mathrm{~m}^{-1}\right)$ and high $\left(\sigma_{m}=1.6 \mathrm{~S} \mathrm{~m}^{-1}\right)$ conductivity media. The transient response of the system can be seen in the time domain plot.

Substituting equation (6) into (10) gives

$$
V_{\text {sys }}(s)=V_{\text {sys } 0}(s)\left(1+2 \sum_{i=1}^{m}(-1)^{i} \mathrm{e}^{-s t_{i}}\right) G_{f}
$$

with $V_{\text {sys } 0}(s)=\frac{b_{1} s^{2}+b_{2} s+b_{3}}{\lambda_{1} s^{4}+\lambda_{2} s^{3}+\lambda_{3} s^{2}+\lambda_{4} s}$ and $G_{f}=\frac{1}{l \kappa}$. Here, $V_{\text {sys } 0}(s)$ is the output response of the system following a unit step excitation signal $U_{0}(t)$ and $G_{f}$ is a scale factor which accounts for the geometry of the system. The expression for $V_{\text {sys } 0}(s)$ can be written in the form of a pole-residue representation:

$$
V_{\mathrm{sys} 0}(s)=\frac{k_{1}}{s-s_{1}}+\frac{k_{2}}{s-s_{2}}+\frac{k_{3}}{s-s_{3}}+\frac{k_{4}}{s-s_{4}}
$$

where $k_{i}$ and $s_{i}(i=1,2,3,4)$ are the residues and poles of $V_{\text {sys } 0}(s)$. Applying the inverse Laplace transform, equation (12) is converted into the time domain:

$$
V_{\text {sys } 0}(t)=k_{1} \mathrm{e}^{s_{1} t}+k_{2} \mathrm{e}^{s_{2} t}+k_{3} \mathrm{e}^{s_{3} t}+k_{4} \mathrm{e}^{s_{4} t} .
$$

Equation (13) is the output response of the system under unit step excitation signal, $U_{0}(t)$, in the time domain. The residues $\left(k_{i}\right)$ and poles $\left(s_{i}\right)$ are dependent on the size of the cell and the dielectric properties of the cell and the medium. These are numerically solved in MATLAB ${ }^{\mathrm{TM}}$ (Mathworks, Inc., USA) for each specific case.According to the time shift property of the Laplace transform, the inverse Laplace transform of equation (11) becomes

$$
V_{\mathrm{sys}}(t)=\left(V_{\mathrm{sys} 0}(t)+2 \sum_{i=1}^{m}(-1)^{i} V_{\mathrm{sys} 0}\left(t-t_{i}\right) U_{0}\left(t-t_{i}\right)\right) G_{f}
$$

This equation is the output response of the system in the continuous time domain.

Simulations of the output response were performed in MATLAB ${ }^{\mathrm{TM}}$, with the following parameters for suspending medium, cell and geometry (figure $2(b)$ and (c)): $R=5 \mu \mathrm{m}$, $d=5 \mathrm{~nm}, \varepsilon_{m}=80 \varepsilon_{o}, \sigma_{m}=1.6 \mathrm{~S} \mathrm{~m}^{-1}, \varepsilon_{\mathrm{mem}}=5 \varepsilon_{o}, \sigma_{\mathrm{mem}}=$ $10^{-8} \mathrm{~S} \mathrm{~m}^{-1}, \varepsilon_{i}=60 \varepsilon_{o}, \sigma_{i}=0.4 \mathrm{~S} \mathrm{~m}^{-1}$ and $h=w=l=$ $20 \mu \mathrm{m}$. These values are used in all the simulations.

Figure 3 shows the simulated output response of the system, $V_{\text {sys }}(t)$, in the time domain for different suspending medium conductivities, non-dimensionalized using the scale 
factor $G_{f}$. The MLS is a current signal; therefore, a lower suspending medium conductivity (higher impedance) gives a greater voltage response. The time domain analysis shows that the MLS technique allows the analysis of the transient response of the system over any time period.

\subsection{Frequency domain analysis}

As stated previously, the impedance spectrum (transfer function) of the system can be characterized in the frequency domain by sequentially applying the FMT and FFT to the response of the system in the time domain - equation (14). To improve the computational efficiency of the FFT, we insert one zero (zero padding) at the end of the output response of the system to perform a $2^{n}$-point FFT instead of $\left(2^{n}-1\right)$ point. The bandwidth of the spectrum obtained using the MLS technique is limited by two parameters: the order of the MLS and the sampling rate. The lowest frequency $f_{\min }$ (which also gives the resolution of the MLS) is determined by the order of the MLS and the sampling rate $f_{s}$ (equation (15a)). The frequency resolution is defined as the difference between two adjacent measured discrete frequencies. Therefore, if the sampling rate is fixed, a higher order MLS gives a higher frequency resolution. From Nyquist sampling theory [56], the highest frequency $f_{\max }$ (equation $(15 b)$ ) is half the sampling rate:

$$
\begin{aligned}
f_{\min } & =\frac{f_{s}}{2^{n}} \\
f_{\max } & =\frac{f_{s}}{2} .
\end{aligned}
$$

Therefore, the number of discrete measurement frequencies is $2^{n-1}$. The data are collected in one sequence period. For example, if $f_{s}=1 \mathrm{MHz}$ and the MLS signal is 10th order, a full data set of 512 frequency points can be collected in approximately $1 \mathrm{~ms}$.

To avoid time aliasing [42], the minimum measurement time needs to be two or more MLS periods with starting the data acquisition from the second MLS. Figure 4(a) shows the simulated impedance spectrum for a cell, using 10thorder MLS with $100 \mathrm{MHz}$ sampling rate, in the frequency band $97.656 \mathrm{kHz}$ to $50 \mathrm{MHz}$ (equation (15)). Variations in the impedance arising from changes in cell parameters are shown (for a fixed cell radius). Around $1 \mathrm{MHz}$, changes in membrane permittivity dominate the impedance, due to interfacial polarization effects - the $\beta$-relaxation [13, 15]. Changes in cytoplasm conductivity are apparent at higher frequencies (above $10 \mathrm{MHz}$ ), where the membrane capacitance is effectively short-circuited.

An important advantage of the MLS technology is high noise immunity. Since the sequence is deterministic and precisely repeatable, the signal-to-noise ratio (SNR) of the system can be improved by synchronous averaging of the output response (prior to FMT) from several sequence periods. This pre-averaging process increases the SNR by $3 \mathrm{~dB}$ for each doubling of the averaging process. To illustrate this, an additive white Gaussian noise (AWGN), $w(t)$, with zero mean and variance $\sigma_{w}^{2}$ was added to the output response of the system. This noise is independent of the input signal, and is representative of the background noise in an (a)

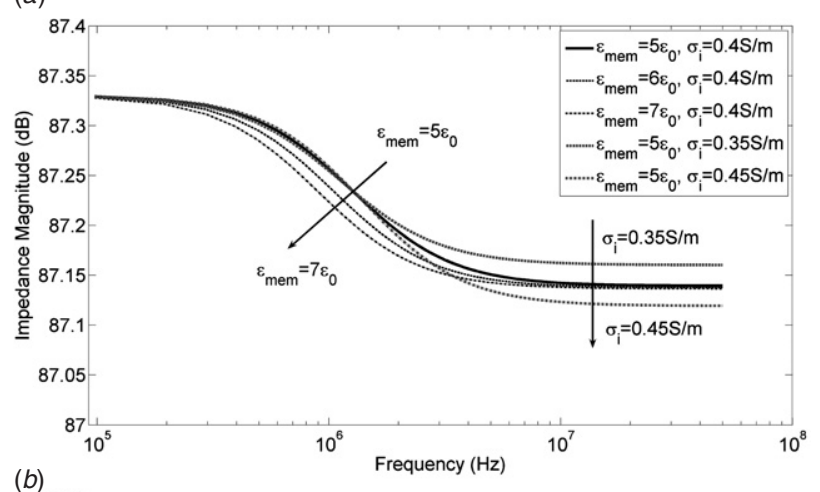

(b)

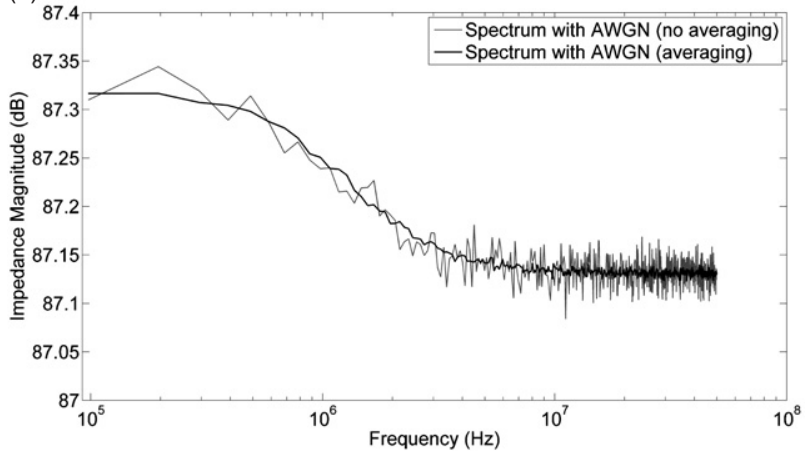

Figure 4. (a) Simulated impedance spectra showing variations due to the different dielectric properties of the cell using MLS analysis. (b) Simulations showing the influence of noise on the impedance spectra (obtained using MLS). Synchronous averaging of the response over several sequences reduces the noise, improving the signal-to-noise ratio (SNR) of the system.

experiment. The output response of the system with AWGN is then $V_{\text {sys } \_n}(t)$ :

$$
V_{\text {sys } \_n}(t)=V_{\text {sys }}(t)+w(t) .
$$

Figure $4(b)$ shows the impedance spectrum of this system with and without averaging. The data are averaged 18 times and the MLS is 10th order, with a sampling rate of $100 \mathrm{MHz}$. The figure clearly shows how the noise signal is attenuated by the process.

In order to examine the accuracy of the impedance spectrum obtained with the MLS, the reference spectrum is taken to be the impedance of a single cell in suspension in the $\omega$-domain (replacing $s$ with $\mathrm{j} \omega$ in equation (7)). The impedance spectrum obtained from MLS technique is by applying the FFT to the impulse response of the system. However, a number of spectral artefacts occur because of FFT sampling errors in both the time and frequency domains. These are the leakage effect, picket-fence effect and aliasing effect [59]. For a fixed sampling rate, the leakage effect and picket-fence effect can be minimized by increasing the number of FFT points, i.e. increasing the length of the MLS. A longer MLS leads to more FFT points, increasing the time window and also gives a higher frequency resolution. The aliasing effect can be minimized by increasing the sampling rate.

Figures 5(a) and (b) show a simulated impedance spectrum, compared to a reference, for two different orders of the MLS (at a fixed sampling rate of $100 \mathrm{MHz}$ ). For 10thorder MLS, there are 1023 elements in a single MLS, while the 14th order has 16383 elements, giving a frequency resolution 


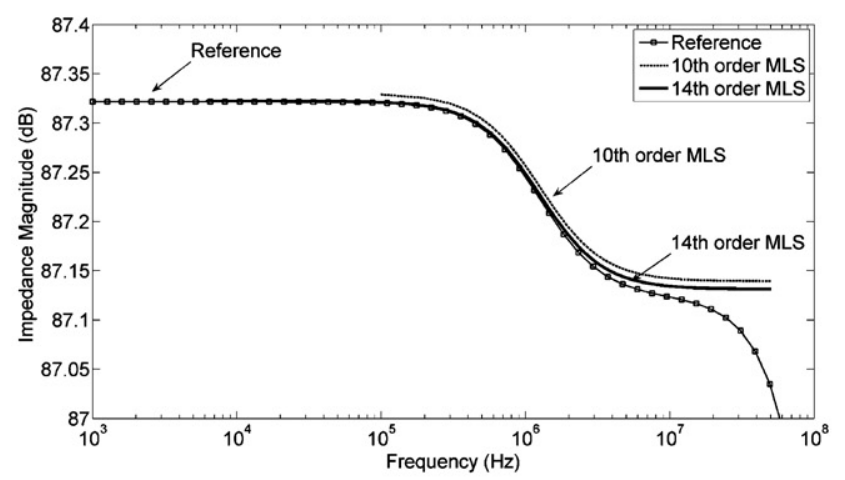

(a)

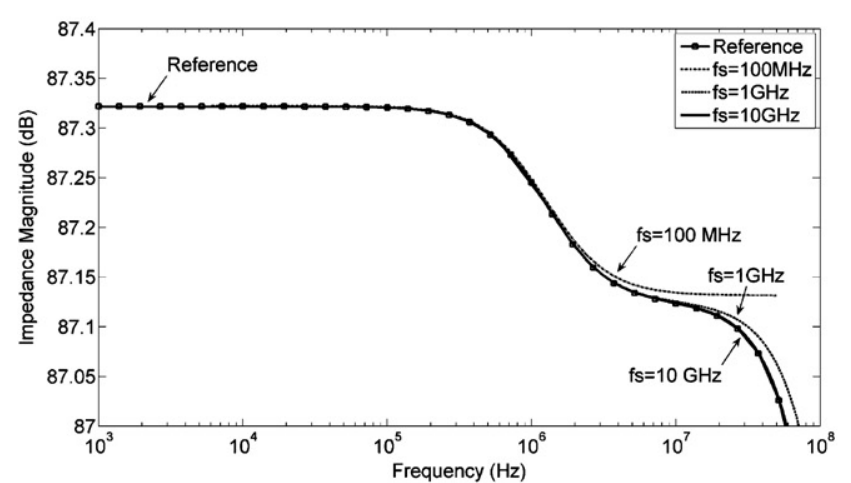

(c)

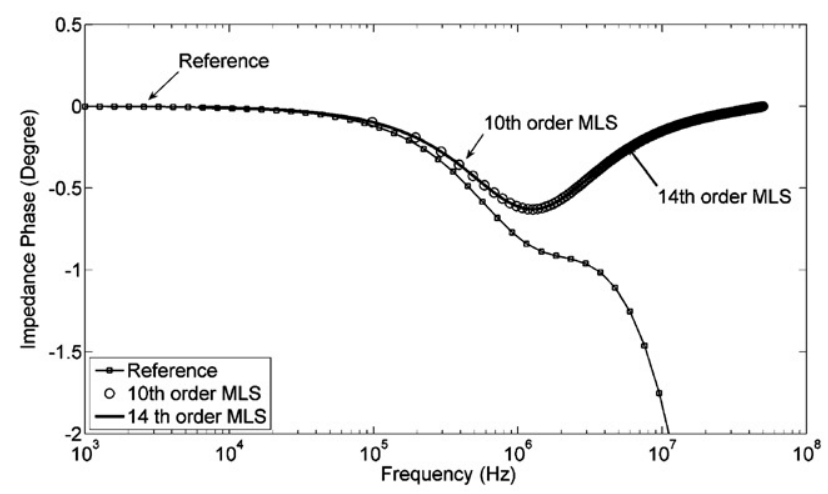

(b)

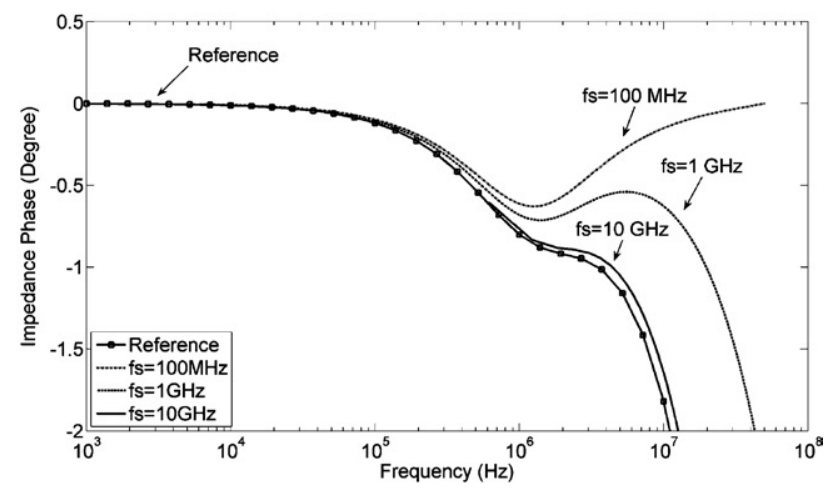

(d)

Figure 5. $(a$ and $b)$ Simulations of the impedance spectra for different orders of the MLS; a longer MLS increases the accuracy. $(c$ and $d$ ) Simulations of the impedance spectra for different sampling rates; higher sampling rate increases the accuracy of the measurement, but restricts the frequency resolution.

of $97.656 \mathrm{kHz}$ for $10 \mathrm{th}$ order and $6.1 \mathrm{kHz}$ for 14 th order, respectively. It can be observed that using a longer MLS leads to a more accurate impedance magnitude spectrum. However, deviations in the phase are unaffected by the order of the MLS, figure $5(b)$, because phase errors are dominated by aliasing effects.

In figures $5(c)$ and $(d)$, the impedance magnitude and phase spectra are compared for different sampling rates, with MLS being set to the 14th order. Increasing the sampling rate leads to much better agreement with the reference spectra for both phase and magnitude. However, for a fixed MLS order, a higher sampling rate reduces the frequency resolution, for example a 14th order MLS with a $100 \mathrm{MHz}$ sampling rate has a frequency resolution of a $6.1 \mathrm{kHz}$, but this changes to $61 \mathrm{kHz}$ for a sampling rate of $1 \mathrm{GHz}$.

In practical measurements, the sampling rate of the system is determined by the speed of the A/D converter in the data acquisition card. Real measurement system cannot achieve the sampling rates (approaching $1 \mathrm{GHz}$ ) used in the theoretical simulations. However, such a high sampling rate is not required for impedance analysis of cells in the frequency range of interest (up to $50 \mathrm{MHz}$ ). The anti-aliasing problem can be solved using a low-pass filter (LPF) prior to A/D conversion, which pre-filters the signal and minimizes the high frequency spectral components. The MLS technique measures the transfer function of the entire system, therefore the impedance information of the cell have to be determined from the transfer-function spectrum. This is discussed in the next section.

\section{Experimental results}

\subsection{MLS cytometer system set-up}

The MLS technology is integrated into a differential ac single frequency impedance measurement system as described previously $[30,36]$. Compared to the 'standard experimental system', fixed frequency ac signal generators and lockin amplifiers are not required. Custom-designed software controls the data flow as shown in figure 2(a). Figure 6 shows how the MLS technology is implemented in the microfluidic cytometer to perform impedance characterization of cells. The MLS signal is generated in software and applied as a voltage to the top pair of electrodes, with the output response measured through the bottom electrodes. A differential amplifier measures the difference between the signals from these two electrodes - the detection and reference volume. This signal is low-pass filtered, sampled and digitized by a data acquisition card (NI 6251, National Instruments Inc., USA) for data processing (FMT, FFT and data selection). This process gives the transfer-function spectrum for the whole system, from which the impedance of a cell is determined. In the measurements, the highest sampling rate used is $1 \mathrm{MHz}$, limited by the NI 6251. A 10th-order MLS was used to provide 'real-time measurement' of flowing cells. The data consisted of 512 discrete spectral components, evenly distributed in the frequency range up to $500 \mathrm{kHz}$ for each period of the MLS (approximately $1 \mathrm{~ms}$ ). 


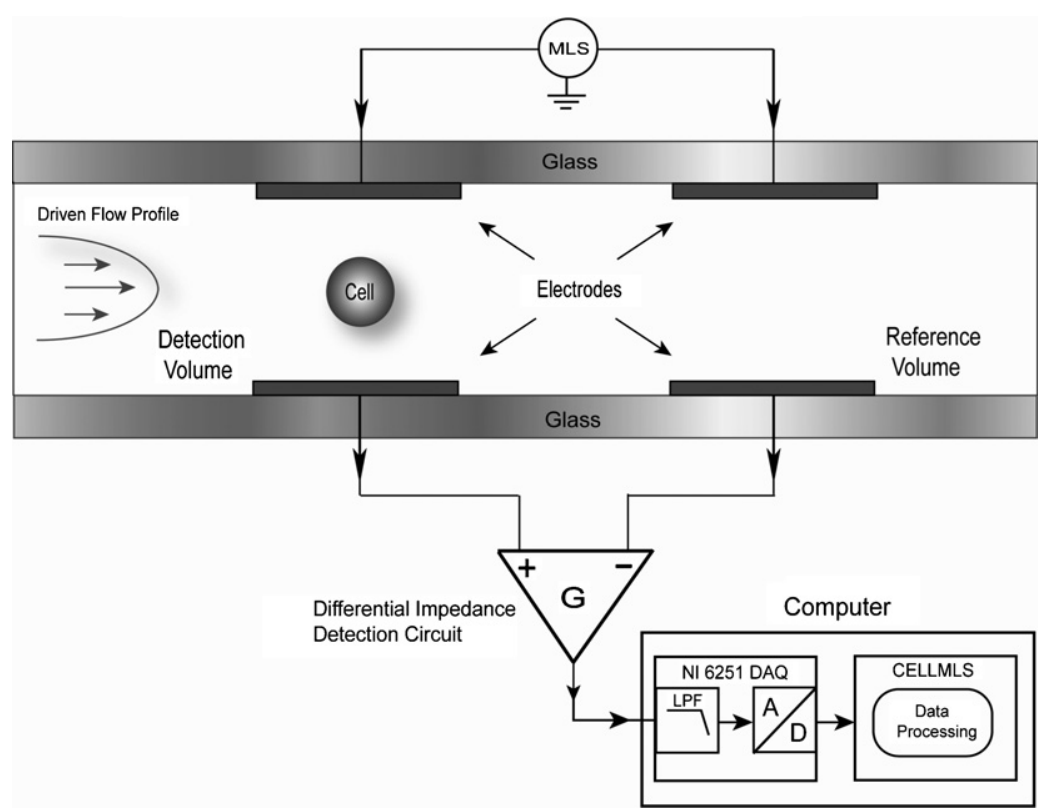

Figure 6. Diagram showing the differential impedance measurement set-up used in the MLS technique.
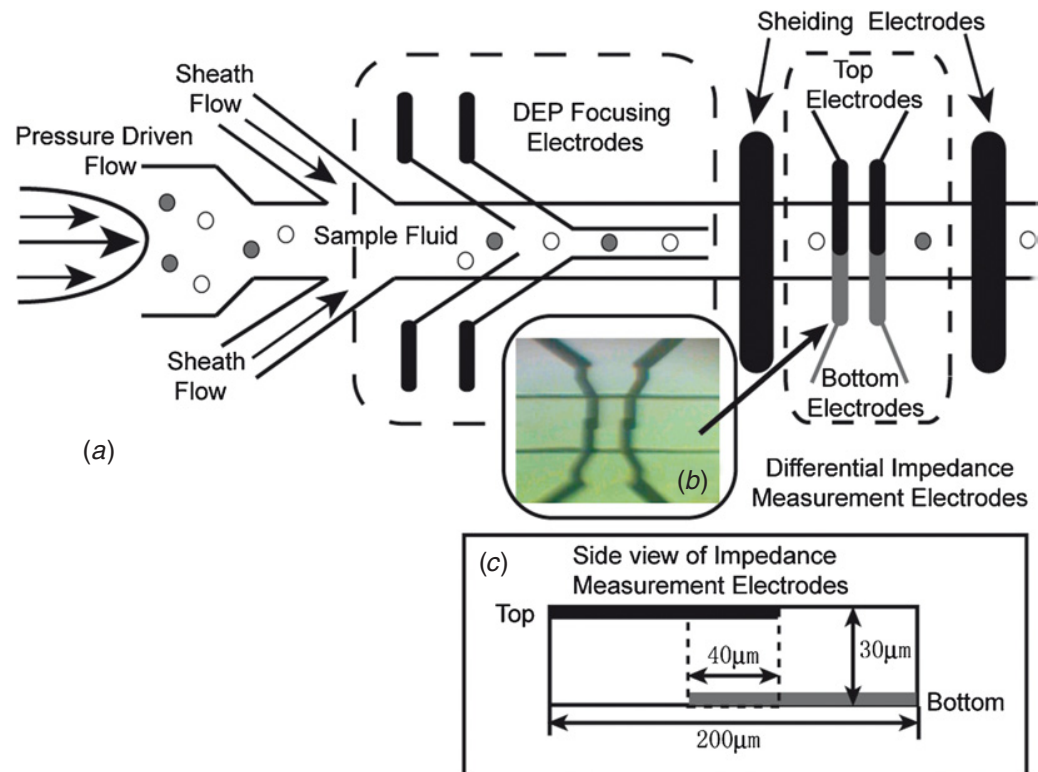

Figure 7. (a) Schematic of the microfluidic chip. (b) A photo of the impedance detection electrodes. (c) A side view of the detection volume.

\subsection{Microfluidic cytometer design}

Figure 7 shows the schematic diagram of the microfluidic chip. Cells flow along a channel $(200 \mu \mathrm{m}$ wide, $30 \mu \mathrm{m}$ high) in which two pairs of overlapping electrodes measure the impedance. This is shown in figure 7(a), with a photograph in figure $7(b)$. The dimension of the sensing region is shown in the inset (figure $7(c)$ ). The cell sample is focused into the central stream either hydrodynamically, using sheath flow, or by dielectrophoresis (DEP), using the two sets of focusing electrodes, as shown in the figure. Two shielding electrodes on either side of the sensing region are grounded to minimize the interactions between the electric fields generated by the DEP electrodes and the impedance detection electrodes.

\subsection{Results and discussions}

Human red blood cells (RBCs) were suspended in phosphate buffered saline (PBS), with a conductivity of $1.6 \mathrm{~S} \mathrm{~m}^{-1}$. The sample solution was pumped through the microchannel, with the flow rate adjusted to obtain the optimum impedance signal. The amplitude of the MLS excitation signal was $0.25 \mathrm{~V}$.

When the impedance in the detection and reference volume (the two pairs of parallel electrodes, figure 6) are balanced, the transfer function of the system is zero. Each time a cell passes through the detection zone, the transfer function is modulated. Figure 8(a) shows an example of the data for single RBC flowing through the detection volume. The data are the real part of the transfer function plotted 

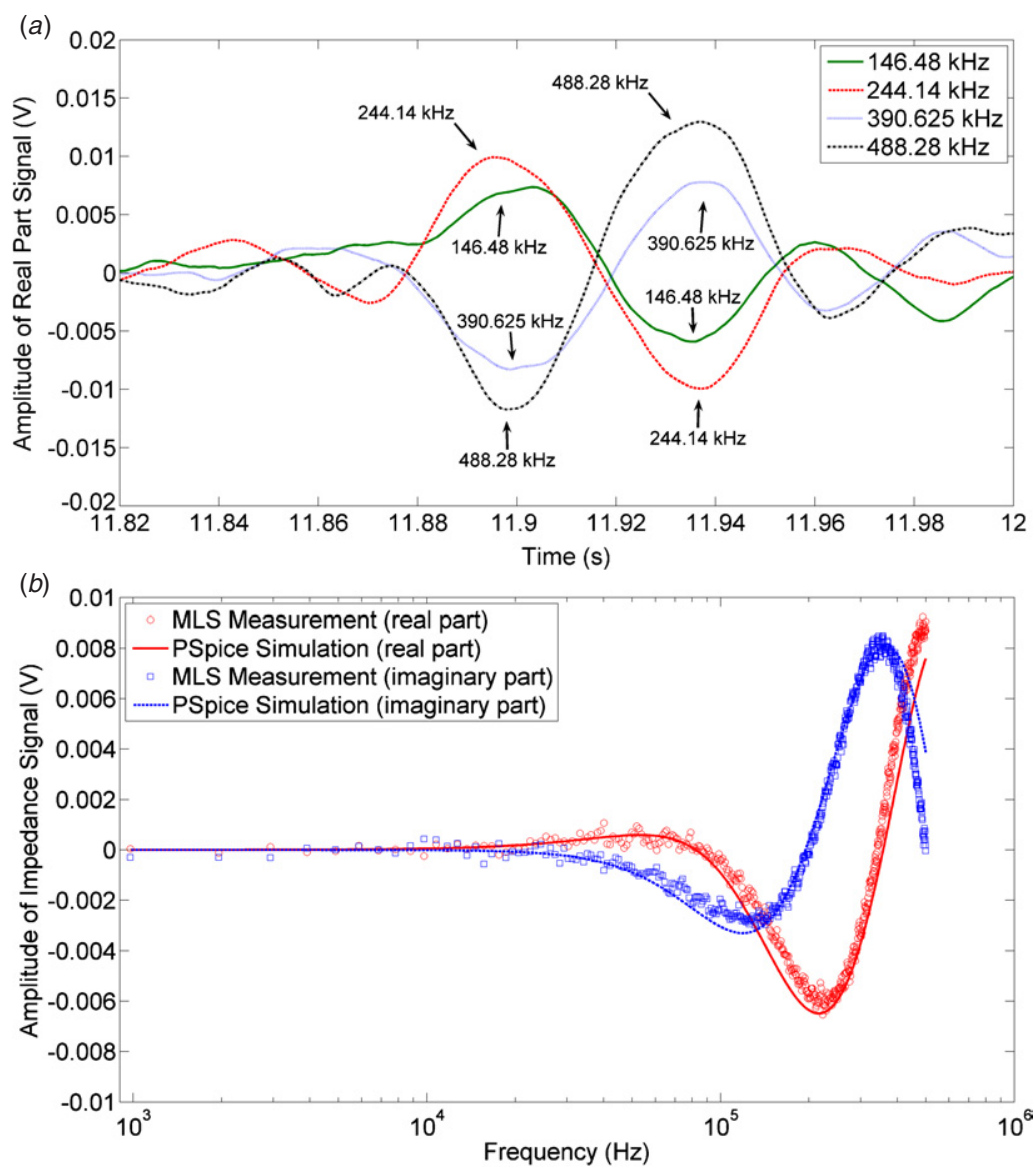

Figure 8. (a) Variation in the real part of the transfer function for a single RBC passing through the detection volume. Four discrete frequencies are plotted: $146.48 \mathrm{kHz}, 244.14 \mathrm{kHz}, 390.625 \mathrm{kHz}$ and $488.28 \mathrm{kHz}$. (b) Real and imaginary parts of the impedance spectrum for a single RBC obtained from PSpice simulation (lines) and MLS measurements (symbols) showing good agreement.

for four discrete frequencies (from 512 total frequencies): $146.48 \mathrm{kHz}, 244.14 \mathrm{kHz}, 390.625 \mathrm{kHz}$ and $488.28 \mathrm{kHz}$. The data were smoothed using a Savitzky-Golay $(\mathrm{S}-\mathrm{G})$ finite impulse response filter and baseline drift corrected by subtracting the averaged background noise level. The figure demonstrates that the MLS can perform multi-frequency measurement of single cells. For 10th-order MLS, 512 individual discrete frequencies are obtained in a single measurement time of approximately $1 \mathrm{~ms}$. The figure shows that the transit time of a single cell across the electrodes is approximately $40 \mathrm{~ms}$. In this time window, 40 periods of the MLS are applied to the cell, giving sufficient time for the cell to be polarized and for data collection. The impedance data for the cell is extracted from the peaks in the data stream, for each discrete frequency.

The MLS measurement results were compared with circuit simulations performed using PSpice (OrCAD Capture, Cadence, Inc., USA). Simulations were performed using the equivalent circuit model for the cell in suspension reported in [30], including the electrical double layer, with a geometric cell constant of 1.106 for the impedance chip. Because the high frequency limit of the present set-up is $500 \mathrm{kHz}$, the simulations and measurements are dominated by the cell size and double layer effects. The MLS technique characterizes the transfer function of the complete system, including the lowpass filter in the data acquisition. This element must therefore be included in the circuit simulations. This was determined by analysing the transfer function of the system without the chip, by connecting the $\mathrm{D} / \mathrm{A}$ and $\mathrm{A} / \mathrm{D}$ ports together using a fixed length of $50 \Omega$ coaxial cable [60]. Figure $8(b)$ compares the impedance obtained from the MLS measurements with PSpice simulations for a single RBC, showing good agreement between the simulation and the measurement.

\section{Conclusion}

A novel impedance spectroscopic technique has been developed with using maximum length sequences. The basic theoretical outline has been described and its application to single cell measurement in a microfluidic cytometer is analysed. With sufficiently long MLS and high enough sampling rate, the complete impedance spectrum of a biological particle can be obtained over a broad frequency band in a short time period. We experimentally demonstrate that the MLS system can be used to measure the complete impedance spectrum of a single red blood cell up to $500 \mathrm{kHz}$ at 512 discrete frequencies. Compared to conventional discrete frequency measurements, the acquisition time of the MLS system is much shorter (typically $1 \mathrm{~ms}$ for a full spectrum). The complexity of the measurement system is substantially reduced and there is a significant saving in hardware instrumentation 
Broadband single cell impedance spectroscopy using maximum length sequences

(no signal generators and lock-in amplifiers). The method enables broadband impedance spectra to be obtained for each cell.

The system described in this paper is still under development and a number of issues remain to be addressed. The modelling assumes that the particle is static in the electric field. However, as shown experimentally, the particle flows through the impedance detection area during data acquisition. Further work is required to develop a dynamic model for a moving particle. Also the bandwidth of the spectrum is limited by the data acquisition rate of the card, which can be extended, typically up to $50 \mathrm{MHz}$ using the latest data acquisition cards. Extension of the frequency range should enable complete dielectric characterization of a single cell, including the membrane, cytoplasm and nucleus.

\section{Acknowledgments}

The work is partly supported by funding from Life Science Initiative of the University of Southampton. The authors would like to thank to Dr Lie Liang Yang for the valuable discussions concerning signal processing theory. Dr Gawad would like to acknowledge funding from the SNSF fellowship.

\section{Appendix}

In equation (7), the coefficients $\lambda_{i}(i=1-4)$ are

$$
\begin{gathered}
\lambda_{1}=\varepsilon_{m} a_{1} \\
\lambda_{2}=\varepsilon_{m} a_{2}+\sigma_{m} a_{1} \\
\lambda_{3}=\varepsilon_{m} a_{3}+\sigma_{m} a_{2} \\
\lambda_{4}=\sigma_{m} a_{3} .
\end{gathered}
$$

And the coefficients $a_{i}$ and $b_{i}(i=1-3)$ are

$$
\begin{aligned}
a_{1}= & \gamma^{3}\left(\varepsilon_{i}+2 \varepsilon_{\mathrm{mem}}\right)\left[\left(\varepsilon_{\mathrm{mem}}+2 \varepsilon_{m}\right)+2 \varphi\left(\varepsilon_{\mathrm{mem}}-\varepsilon_{m}\right)\right] \\
& +2\left(\varepsilon_{i}-\varepsilon_{\mathrm{mem}}\right)\left[\left(\varepsilon_{\mathrm{mem}}-\varepsilon_{m}\right)+\varphi\left(2 \varepsilon_{\mathrm{mem}}+\varepsilon_{m}\right)\right] \\
a_{2}= & \gamma^{3}\left(\varepsilon_{i}+2 \varepsilon_{\mathrm{mem}}\right)\left[\left(\sigma_{\mathrm{mem}}+2 \sigma_{m}\right)+2 \varphi\left(\sigma_{\mathrm{mem}}-\sigma_{m}\right)\right] \\
& +\gamma^{3}\left(\sigma_{i}+2 \sigma_{\mathrm{mem}}\right)\left[\left(\varepsilon_{\mathrm{mem}}+2 \varepsilon_{m}\right)+2 \varphi\left(\varepsilon_{\mathrm{mem}}-\varepsilon_{m}\right)\right] \\
& +2\left(\varepsilon_{i}-\varepsilon_{\mathrm{mem}}\right)\left[\left(\sigma_{\mathrm{mem}}-\sigma_{m}\right)+\varphi\left(2 \sigma_{\mathrm{mem}}+\sigma_{m}\right)\right] \\
& +2\left(\sigma_{i}-\sigma_{\mathrm{mem}}\right)\left[\left(\varepsilon_{\mathrm{mem}}-\varepsilon_{m}\right)+\varphi\left(2 \varepsilon_{\mathrm{mem}}+\varepsilon_{m}\right)\right] \\
a_{3}= & \gamma^{3}\left(\sigma_{i}+2 \sigma_{\mathrm{mem}}\right)\left[\sigma_{\mathrm{mem}}(1+2 \varphi)+2 \sigma_{m}(1-\varphi)\right] \\
& +2\left(\sigma_{i}-\sigma_{\mathrm{mem}}\right)\left[\sigma_{\mathrm{mem}}(1+2 \varphi)-\sigma_{m}(1-\varphi)\right] \\
b_{1}= & \gamma^{3}\left(\varepsilon_{i}+2 \varepsilon_{\mathrm{mem}}\right)\left[\left(\varepsilon_{\mathrm{mem}}+2 \varepsilon_{m}\right)-\varphi\left(\varepsilon_{\mathrm{mem}}-\varepsilon_{m}\right)\right] \\
& +\left(\varepsilon_{i}-\varepsilon_{\mathrm{mem}}\right)\left[2\left(\varepsilon_{\mathrm{mem}}-\varepsilon_{m}\right)-\varphi\left(2 \varepsilon_{\mathrm{mem}}+\varepsilon_{m}\right)\right] \\
b_{2}= & \gamma^{3}\left(\varepsilon_{i}+2 \varepsilon_{\mathrm{mem}}\right)\left[\left(\sigma_{\mathrm{mem}}+2 \sigma_{m}\right)-\varphi\left(\sigma_{\mathrm{mem}}-\sigma_{m}\right)\right] \\
& +\gamma^{3}\left(\sigma_{i}+2 \sigma_{\mathrm{mem}}\right)\left[\left(\varepsilon_{\mathrm{mem}}+2 \varepsilon_{m}\right)-\varphi\left(\varepsilon_{\mathrm{mem}}-\varepsilon_{m}\right)\right] \\
& +\left(\varepsilon_{i}-\varepsilon_{\mathrm{mem}}\right)\left[2\left(\sigma_{\mathrm{mem}}-\sigma_{m}\right)-\varphi\left(2 \sigma_{\mathrm{mem}}+\sigma_{m}\right)\right] \\
& +\left(\sigma_{i}-\sigma_{\mathrm{mem}}\right)\left[2\left(\varepsilon_{\mathrm{mem}}-\varepsilon_{m}\right)-\varphi\left(2 \varepsilon_{\mathrm{mem}}+\varepsilon_{m}\right)\right] \\
b_{3}= & \gamma^{3}\left(\sigma_{i}+2 \sigma_{\mathrm{mem}}\right)\left[\sigma_{\mathrm{mem}}(1-\varphi)+\sigma_{m}(2+\varphi)\right] \\
& +\left(\sigma_{i}-\sigma_{\mathrm{mem}}\right)\left[2 \sigma_{\mathrm{mem}}(1-\varphi)-\sigma_{m}(2+\varphi)\right]
\end{aligned}
$$

where $\varphi$ is the volume fraction and $\gamma=(R+d) / d$ is the ratio of the outer and inner layers of the particle. For the detailed derivations, see [55].

\section{References}

[1] Höber R 1910 Eine Methode die elektrische Leitfaehigkeit im Innern von Zellenzu messen Arch. Ges. Physiol. $133237-59$

[2] Höber R 1912 Ein zweites Verfahren die Leitfaehigkeit im Innern von Zellen ze messem Arch. Ges. Physiol. 148 189-221

[3] Höber R 1913 Messungen der inneren Leitfaehigkeit von Zelen III Arch. Ges. Physiol. 150 15-45

[4] Fricke H 1924 A mathematical treatment of the electric conductivity and capacity of disperse systems: I. The electric conductivity of a suspension of homogeneous spheroids Phys. Rev. 24 575-87

[5] Fricke H 1925 A mathematical treatment of the electric conductivity and capacity of disperse systems: II. The capacity of a suspension of conducting membrane for a current of low frequency Phys. Rev. 26 678-81

[6] Fricke H 1931 The electric conductivity and capacity of disperse systems Physics 1 106-15

[7] Fricke $H$ and Curtis H J 1935 The electric impedance of hemolyzed suspensions of mammalian erythrocytes J. Gen. Physiol. 18 821-36

[8] Cole K S 1928 Electric impedance of suspensions of spheres J. Gen. Physiol. 12 29-36

[9] Cole K S 1928 Electric impedance of suspensions of Arbacia eggs J. Gen. Physiol. 12 37-54

[10] Cole K S 1935 Electric impedance of Hipponoë eggs J. Gen. Physiol. 18 877-87

[11] Cole K S and Cole R H 1936 Electric impedance of Asteria eggs J. Gen. Physiol. 19 609-23

[12] Cole K S and Cole R H 1936 Electric impedance of Arbacia eggs J. Gen. Physiol. 19 625-32

[13] Schwan H P 1957 Electrical properties of tissue and cell suspensions Adv. Biol. Med. Phys. 5 147-209

[14] Schwan H P 1963 Determination of biological impedances Phys. Tech. Bio. Res. vol 6, ed W L Nastuk (New York: Academic) pp 323-406

[15] Foster K R and Schwan H P 1989 Dielectric properties of tissues and biological materials: a critical review Crit. Rev. Biomed. Eng. 17 25-104

[16] Pethig R and Kell D B 1987 The passive electrical properties of biological systems: their significance in physiology, biophysics and biotechnology Phys. Med. Biol. 32 933-70

[17] Zimmermann U, Friedrich U, Mussauer H, Gessner P, Hämel K and Sukhorukov V 2000 Electromanipulation of mammalian cells: fundamentals and application IEEE Trans. Plasma Sci. 28 72-82

[18] Hanai T, Asami K and Koizumi N 1979 Dielectric theory of concentrated suspensions of shell-spheres in particular reference to the analysis of biological cell suspensions Bull. Inst. Chem. Res. Kyoto Univ. 57 297-305

[19] Asami K 2002 Characterization of heterogeneous systems by dielectric spectroscopy Prog. Polym. Sci. 27 1617-59

[20] Pethig R, Huang Y, Wang X-B and Burt J P H 1992 Positive and negative dielectrophoretic collection of colloidal particles using interdigitated castellated microelectrodes J. Phys. D: Appl. Phys. 24 881-8

[21] Holmes D and Morgan H 2002 Cell positioning and sorting using dielectrophoresis Eur. Cells Mater. 4 120-22

[22] Voldman J 2006 Electrical forces for microscale cell manipulation Ann. Rev. Biomed. Eng. 8 425-54

[23] Huang Y, Wang X-B, Tame J A and Pethig R 1993 Electrokinetic behaviour of colloidal particles in travelling electric field: studies using yeast cells J. Phys. D: Appl. Phys. 26 1528-35

[24] Cui L, Holmes D and Morgan H 2001 The dielectrophoretic levitation and separation of latex beads in microchips Elelctrophoresis 22 3893-901

[25] Hölzel R 1997 Electrorotation of single yeast cells at frequencies between $100 \mathrm{~Hz}$ and $1.6 \mathrm{GHz}$ Biophys. $J$. 73 1103-9 
[26] Dalton C, Goater A D, Burt J P H and Smith H V 2004 Analysis of parasites by electrorotation J. Appl. Microbiol. $9624-32$

[27] Suehiro J, Yatsunami R, Hamada R and Hara M 1999 Quantitative estimation of biological cell concentration suspended on aqueous medium by using dielectrophoretic impedance measurement method J. Phys. D: Appl. Phys. 32 2814-20

[28] Ayliffe H E, Frazier A B and Rabbit R D 1999 Electric impedance spectroscopy using microchannels with integrated metal electrodes IEEE Microelectromech. Syst. $850-7$

[29] Sohn L L, Saleh O A, Facer G R, Beavis A J, Allan R S and Notterman D A 2000 Capacitance cytometry: measuring biological cells one by one Proc. Natl Acad. Sci. 97 10687-90

[30] Morgan H, Sun T, Holmes D, Gawad S and Green N G 2007 Single cell dielectric spectroscopy J. Phys. D: Appl. Phys. 40 61-70

[31] Feldman Yu, Ermolina I and Hayashi Y 2003 Time domain dielectric spectroscopy study of biological systems IEEE Trans. Dielectr. Electr. Insul. 10 728-53

[32] Gawad S, Schild L and Renaud Ph 2001 Micromachined impedance spectroscopy flow cytometer for cell analysis and particle sizing Lab Chip 1 76-82

[33] Gascoyne P R C, Anderson T, Vykoukal J and Becker F 2004 Particle impedance sensor US Patent 6703819

[34] Gawad S, Cheung K, Seger U, Bertsch A and Renaud Ph 2004 Dielectric spectroscopy in a micromachined flow cytometer: theoretical and practical considerations Lab Chip 4 241-51

[35] Cheung K, Gawad S and Renaud Ph 2005 Impedance spectroscopy flow cytometer: on-chip label-free cell differentiation Cytometry A 65 124-32

[36] Morgan H, Holmes D and Green N G 2006 High speed simultaneous single particle impedance and fluorescence analysis on a chip Curr. Appl. Phys. 6 367-70

[37] Davies W D T 1966 Generation and properties of maximum-length sequences Control 302-4

[38] Cohn M and Lempel A 1977 On fast M-sequence transforms IEEE. Trans. Inform. Theory 135-7

[39] Schroeder M R 1979 Integrated-impulse method measuring sound decay without using impulses J. Acoust. Soc. Am. $66497-500$

[40] Borish J and Angell J B 1983 An efficient algorithm for measuring the impulse response using pseudorandom noise J. Audio Eng. Soc. 31 478-88

[41] Borish J 1985 Self-contained crosscorrelation program for maximum-length sequences J. Audio Eng. Soc. 33 888-91

[42] Rife D D and Vanderkooy J 1989 Transfer-function measurement with maximum-length sequences $J$. Audio Eng. Soc. 37 419-44
[43] Xiang N 1992 Using M-sequences for determining the impulse responses of LTI-systems Signal Process. 28 139-52

[44] Vanderkooy J 1994 Aspect of MLS measuring systems J. Audio Eng. Soc. 42 219-31

[45] Vorlander M and Kob M 1997 Practical aspects of MLS measurements in building acoustics Appl. Acoust. 52 239-58

[46] Ciric D G 1999 Sychronisation and excitation for MLS technique implementation in room acoustics TELSIKS' 99 653-6

[47] Xiang N and Schroeder M R 2003 Reciprocal maximum-length sequence pairs for acoustical dual source measurements J. Acoust. Soc. Am. 113 2754-61

[48] Xiang N, Daigle J N and Kleiner M 2005 Simultaneous acoustic channel measurement via maximal-length-related sequences J. Acoust. Soc. Am. 117 1889-94

[49] Weckström M, Kouvalainen E and Juusola M 1992 Measurement of cell impedance in frequency domain using discontinuous current clamp and white-noise-modulated current injection Pfügers Arch. 421 469-72

[50] Schneider I 1996 Broadband signals for electrical impedance measurements of long bone fractures Proc. 18th Annual Int Conf. of the IEEE EMBS pp 1934-5

[51] Amrani M E H, Dowdeswell R M, Payne P A and Persaud K C 1998 Pseudo-random binary sequence interrogation technique for gas sensors Sensors Actuators B 47 118-24

[52] Rufer L, Mir S, Simeu E and Domingues C 2003 On-chip testing of MEMS using pseudo-random test sequences, DTIP of MEMS/MOEMS pp 50-5

[53] Xiang N and Sabatier J M 2004 Laser Doppler vibrometer-based acoustic landmine detection using the fast M-sequence transform IEEE Geosci. Remote Sens. Lett. $1292-4$

[54] Xiang N and Chu D Z 2004 Fast M-sequence transform for quasi-backscatter sonar in fisheries and zooplankton survey applications Proc. ICSP pp 2433-6

[55] Sun T, Gawad S, Green N G and Morgan H 2007 Dielectric spectroscopy of single cells: time domain analysis using Maxwell's mixture equation J. Phys. D: Appl. Phys. 40 1-8

[56] Oppenheim A V, Willsky A S and Young I T 1983 Signal Systems (London: Prentice-Hall)

[57] Maxwell J C 1954 A Treatise on Electricity and Magnetism (New York: Dover)

[58] Sun T, Green N G, Gawad S and Morgan H 2007 Analytical electric field and sensitivity analysis for two microfluidic impedance cytometer designs IET Nanobiotechnol. at press

[59] Ziemer R E, Tranter W H and Fannin D R 1998 Signals \& Systems 4th edn (New York: Prentice-Hall)

[60] Gawad S, Sun T, Green N G and Morgan H 2007 Impedance spectroscopy using maximum length sequences: application to single cell analysis Rev. Sci. Instrum. 78054301 